\title{
Influence of Molecular Aggregation on Electron Transfer at the Perylene Dimide/Indium-Tin Oxide Interface
}

Yilong Zheng, ${ }^{1, \dagger}$ Fadi M. Jradi, ${ }^{2, \dagger}$ Timothy C. Parker, ${ }^{2}$ Stephen Barlow, ${ }^{2}$ Seth R. Marder, ${ }^{2, *}$ S. Scott Saavedra ${ }^{1, *}$

${ }^{1}$ Department of Chemistry \& Biochemistry, University of Arizona, Tucson, AZ 85721-00041

${ }^{2}$ School of Chemistry \& Biochemistry and the Center for Organic Photonics and Electronics, Georgia Institute of Technology, Atlanta, GA 30332-0400

${ }^{\dagger}$ The authors declare that $\mathrm{YZ}$ and FMJ have contributed equally to this body of work

* Contact author: saavedra@email.arizona.edu 


\section{ABSTRACT}

Chemisorption of an organic monolayer to tune the surface properties of a transparent conductive oxide (TCO) electrode can improve the performance of organic electronic devices that rely on efficient charge transfer between an organic active layer and a TCO contact. Here a series of perylene diimides (PDIs) was synthesized and used to study relationships between monolayer structure/properties and electron transfer kinetics at PDI-modified indium tin oxide (ITO) electrodes. In these PDI molecules, one of the imide substituents is a benzene ring bearing a phosphonic acid (PA) and the other is a bulky aryl group that is twisted out of the plane of the PDI core. The size of the bulky aryl group and the substitution of the benzene ring bearing the PA were both varied which altered the extent of aggregation when these molecules were absorbed as monolayer films (MLs) on ITO, as revealed by both attenuated total reflectance (ATR) and total internal reflection fluorescence (TIRF) spectra. Polarized ATR measurements indicate that in these MLs, the long axis of the PDI core is tilted at an angle of $33^{\circ}-42^{\circ}$ relative to the surface normal; the tilt angle increased as the degree of bulky substitution increased. Rate constants for electron transfer $\left(k_{\mathrm{s}, \mathrm{opt}}\right)$ between these redox-active modifiers and ITO were determined by potential-modulated ATR spectroscopy. As the degree of PDI aggregation was reduced, $k_{\mathrm{s}, \mathrm{opt}}$ declined which is attributed to a reduction in the lateral electron self-exchange rate between adsorbed PDI molecules, as well as the heterogeneous conductivity of the ITO electrode surface. Photoelectrochemical measurements using a dissolved aluminum phthalocyanine as an electron donor showed that ITO modified with any of these PDIs is a more effective electron-collecting electrode than bare ITO.

Keywords: perylene diimide, phosphonic acid, electrochemistry, electron transfer, indium-tin oxide, 
organic electronics, electron self-exchange, potential-modulated ATR spectroscopy

\section{INTRODUCTION}

Transparent conductive oxides (TCOs) are essential electrode materials for applications in organic electronics, such as organic photovoltaics $(\mathrm{OPVs}),{ }^{1-4}$ organic thin-film transistors, ${ }^{5}$ organic light-emitting diodes, ${ }^{6,7}$ and perovskite solar cells. ${ }^{8}$ Understanding and controlling the surface properties of TCO electrodes can improve the performance of these types of devices. ${ }^{2,47}$ As a consequence, strategies to modify TCOs have been developed, such as chemisorption of an organic monolayer to tune the TCO surface composition, ${ }^{3,9,10}$ surface free energy, ${ }^{1,2,4,11-13}$ work function, ${ }^{1,2,11-13}$ and chemical stability. ${ }^{6,14}$ For example, Khodabakhsh et al. ${ }^{2}$ reported that the power conversion efficiency (PCE) of a $\mathrm{CuPc}: \mathrm{C}_{60}$ (copper phthalocyanine:fullerene) heterojunction solar cell increases to $1.27 \%$ when the indium tin oxide (ITO) contact is modified with 4-chloro-benzoyl-chloride, compared with $0.16 \%$ using non-modified ITO and $0.81 \%$ using PEDOT:PSS-modified ITO. The enhancement was attributed to a greater short circuit current $\left(J_{\mathrm{SC}}\right)$, courtesy of an enhanced interfacial electron-transfer $(\mathrm{ET})$ rate at the anode resulting from a shift in the ITO work function, which lowered the injection barrier between the electrode Fermi level and the CuPc HOMO. ${ }^{2}$

Redox-active modifiers offer the possibility of further enhancing the charge-collection efficiency at a TCO/organic interface by serving as a "mediator" layer with a tunable redox potential that can be matched to the relevant charge-transport energy levels. ${ }^{3,15}$ Lin et al. ${ }^{15}$ used redox-active zinc phthalocyanine phosphonic acids (ZnPcPAs) to modify ITO electrodes that were tested as bottom contacts in $\mathrm{CuPc} / \mathrm{C}_{60}$ planar heterojunction solar cells. ZnPcPA-modified cells outperformed cells 
prepared using ITO modified with redox-inactive alkyl phosphonic acids. ${ }^{15}$ Song et al. ${ }^{4}$ showed that diarylamine chlorosilane $\left(\mathrm{Ar}_{2} \mathrm{~N}-\left(\mathrm{CH}_{2}\right)_{\mathrm{n}}-\mathrm{SiCl}_{3}, \mathrm{Ar}=3,4\right.$-difluorophenyl) redox-active modifiers can serve as a hole-selective interfacial layer on an ITO electrode in bulk-heterojunction OPVs; the PCE of these devices was slightly higher than that of devices in which PEDOT:PSS was utilized as the interfacial layer. ${ }^{4}$ These and other papers ${ }^{15-21}$ have demonstrated that the molecular design of a redox-active modifier, such as the type, position and length of its bridging moiety and the presence of solubilizing groups or bulky substituents, may significantly affect the charge-collection kinetics and efficiency at modified TCO surfaces, because these structural parameters dictate properties such as molecular orientation, tunneling distance, degree of aggregation, and/or interface dipole magnitude and orientation.

The solid-state electron affinities (EA) and solution reduction potentials of perylene-3,4,9,10-tetracarboxylic acid diimides: (perylene diimides, PDIs) are well matched to those of some acceptor materials (such as fullerenes) commonly used in organic electronic devices, ${ }^{22-24}$ suggesting their use as redox-active modifiers in these devices. Furthermore, PDI redox potentials can be tuned, most effectively through substitutions at the "bay" $(1,6,7,12)$ and/or "ortho" $(2,5,8,11)$ positions, allowing them to be energetically matched to different active-layer and TCO materials. ${ }^{25,26}$ In a recent study, ${ }^{27}$ we began examining the properties of PDI monolayers tethered to ITO via phosphonic acid (PA) anchoring groups. ET rate constants exceeding $10^{3} \mathrm{~s}^{-1}$ were measured for PDI-PA monolayers on ITO, and a dependence on the bridge length, which dictated the tunneling distance, was demonstrated. The UV-visible absorption spectra of these films showed that the PDI molecules were highly aggregated.

Here we report an assessment of the effects of aggregation on ET behavior, for which a series of 
new asymmetric PDI-PA modifiers was synthesized and characterized. The new molecules, (phenyl) $)_{2}$-PDI-PA, (terphenyl) $)_{2}$-PDI-PA and (terphenyl) $)_{2}$-PDI-IP-PA, are shown in Figure 1, along with the previously described PDI-phenyl-PA ${ }^{27}$ (which in this paper is named alkyl-PDI-PA). These molecules are designed to have different degrees of aggregation when deposited as a monolayer film on an ITO electrode. Replacing the C11 chains on alkyl-PDI-PA with phenyl and terphenyl substituents that are necessarily twisted out of the plane of the perylene core is expected to reduce the intermolecular interactions in (phenyl) $)_{2}$-PDI-PA and (terphenyl) $)_{2}$-PDI-PA films (see space-filling models in Figure S1). (Terphenyl) $)_{2}$-PDI-IP-PA differs from (terphenyl) $)_{2}$-PDI-PA in the presence of diisopropyl substitution of the phenylene group that links the PDI to the PA binding group; this modification is expected to twist the bridging group out of the plane of the perylene core, further reducing intermolecular interactions. Although aggregation can be controlled by substitution at other positions, ${ }^{28,29}$ the LUMO is characterized by a nodal plane that passes through the imide nitrogen atoms; ${ }^{25}$ thus, in the absence of strong inductive effects, modifications can be made at these positions without simultaneously altering the energy levels of the PDI.

Monolayers of these molecules adsorbed on ITO were characterized using a variety of techniques to assess relationships between film structure and ET kinetics. As the number and size of the substituents on the PDI modifier increased, both the degree of aggregation and the ET rate constant decreased; the latter result is attributed to a lower rate of electron self-exchange between adsorbed molecules. Overall, these results provide guidance for further development of redox-active adsorbates designed to modulate charge transfer at TCO contacts in organic electronic devices. 


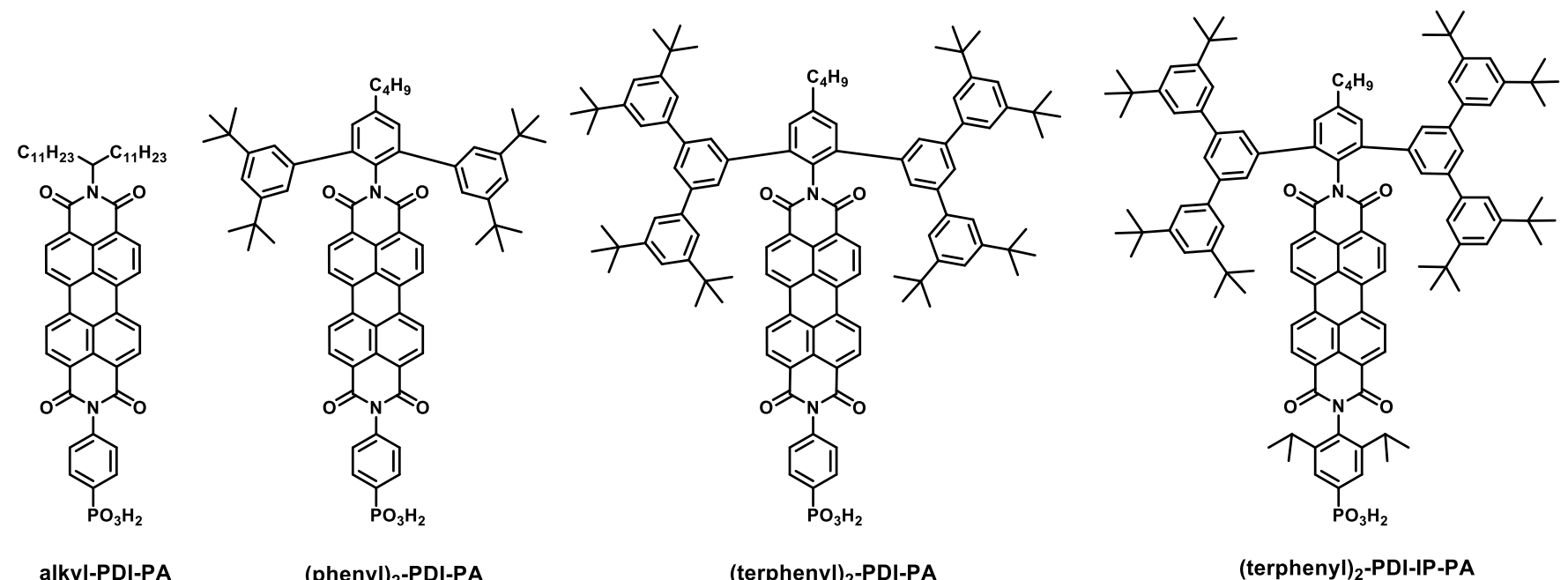

Figure 1. The four PDI-PA molecules used in this study with different imide substituents to control the degree of aggregation.

\section{EXPERIMENTAL SECTION}

\section{Preparation of PDI films on ITO}

ITO on Schott BoroFloat glass with a layer thickness of $c a .150 \mathrm{~nm}$ and a sheet resistance of 20-30 $\Omega \cdot \mathrm{cm}^{-2}$ was purchased from Thin Film Devices. ITO sheets were cut into 1 in. $\times 3$ in. slides, for ATR and TIRF experiments, or $1 / 2$ in. $\times 1 / 2$ in. slides for electrochemical experiments. ITO slides were cleaned, stored, and activated as described previously. ${ }^{27}$

PDI-PA molecules were synthesized, as outlined in Scheme 1, with the detailed procedures and relevant spectra presented in the Supporting Information (SI). Alkyl-PDI-PA films were prepared by adsorption from solution using the same protocol described in the previous paper. ${ }^{27}$ To prepare (phenyl) $)_{2}$-PDI-PA, (terphenyl) $)_{2}$-PDI-PA and (terphenyl) $)_{2}$-PDI-IP-PA films, activated ITO slides were immersed into a $20 \mu \mathrm{M}$ solution of the respective molecule in tetrahydrofuran (THF) at room 
temperature for $2 \mathrm{~h}$, then rinsed with a copious amount of THF and dried with either flowing $\mathrm{N}_{2}$ (for electrochemical measurements) or in air (for ATR measurements) before characterization.

\section{Fluorescence emission spectroscopy}

Fluorescence emission measurements were performed with a custom-built, total internal reflection fluorescence (TIRF) microscope assembled around a Nikon Diaphot. The $488 \mathrm{~nm}$ line of an argon ion laser (5500AWC-00C, Ion Laser Technology) was directed through a half wave Fresnel rhomb (Karl Lambrecht, MFRC-10-UN-580,) to select transverse magnetic (TM) polarized excitation. The beam was coupled into the internal reflection element (IRE) mounted in a flow cell using a BK7 prism. The IRE was an ITO slide with an adsorbed PDI film. Fluorescence emission was collected using a 10x objective and passed through a $620 \pm 90 \mathrm{~nm}$ bandpass filter (Omega Optical), then coupled into a monochromator (Spectra Pro 275, Acton Research) and detected using a PMT (Thorn EMI 9816B; Products for Research TE104RF housing and S504 power supply) and a photon counter (Stanford Research Systems SR400). Immediately after each measurement on a PDI-PA film, a dextran-fluorescein dye solution ( $c a .5 \mathrm{mM}$ in water) was injected into the flow cell and the fluorescence intensity was measured. This procedure was performed to account for differences in excitation power and collection efficiency, allowing the spectra of PDI-PA films measured in separate experiments to be quantitatively compared. 
Scheme 1. Overview of the perylene diimide synthesis. ${ }^{*}$
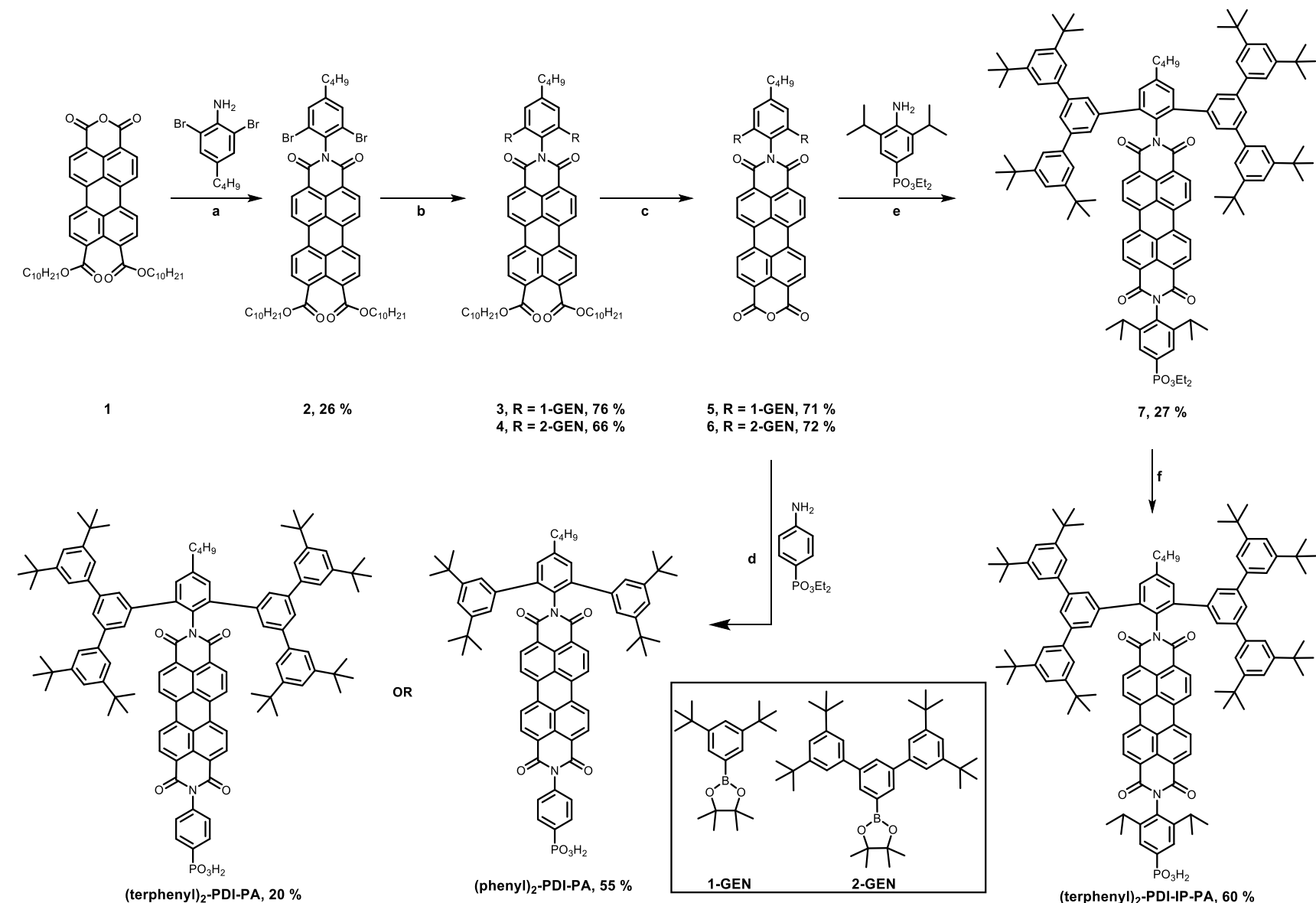

$5, R=1-G E N, 71 \%$
$6, R=2-G E N, 72 \%$

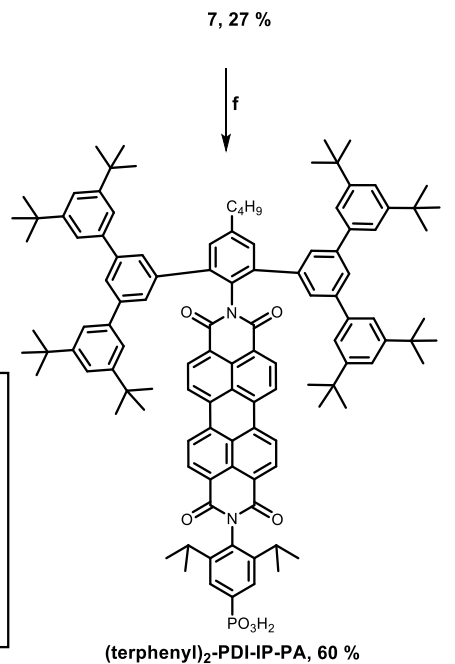

* (a) 4-Dimethylaminopyridine, imidazole/toluene, $140{ }^{\circ} \mathrm{C}$, overnight. (b) $\mathrm{PdCl}_{2}$ (dppf). $\mathrm{CH}_{2} \mathrm{Cl}_{2}$, toluene, methanol, 1-GEN or

2-GEN. (c) i) Toluene, $95{ }^{\circ} \mathrm{C}$; ii) p-toluenesulfonic acid, $95{ }^{\circ} \mathrm{C}, 5$ hours. (d) i) 5 or $\mathbf{6}$, aniline, zinc acetate, imidazole/toluene,

$140{ }^{\circ} \mathrm{C}, 3$ hours; ii) bromotrimethylsilane, dichloromethane, room temperature, 24-72 hours. (e) i) 6, aniline, propanoic acid,

$160{ }^{\circ} \mathrm{C}$, overnight. (f) (i) Bromotrimethyl silane, dichloromethane, room temperature, overnight; ii) water/THF with a few drops of hydrochloric acid. 


\section{Cyclic voltammetry}

Cyclic voltammetry (CV) was performed with a standard three-electrode configuration as described previously. ${ }^{27}$ The nonaqueous $\mathrm{Ag} / \mathrm{AgNO}_{3}(0.01 \mathrm{M})$ pseudo-reference electrode was calibrated with the ferrocene/ferrocenium $\left(\mathrm{Fc} / \mathrm{Fc}^{+}\right)$redox couple; the midpoint potential was measured to be $0.11 \mathrm{~V}$ which was assumed to be $-5.1 \mathrm{eV}$ vs. vacuum. ${ }^{30}$ Tetrabutylammonium perchlorate (TBAP) solution $(0.1 \mathrm{M})$ in acetonitrile was used as the supporting electrolyte; it was deoxygenated with a gentle flow of Ar for $1 \mathrm{~h}$ inside the fully assembled electrochemical cell in a glove bag, also filled with Ar. All CV measurements were performed immediately after deoxygenation to minimize the effect of dissolved $\mathrm{O}_{2}$. The PDI-PAs are insoluble in acetonitrile, which eliminated the possibility of desorption during the electrochemical measurements.

\section{ATR spectroscopy}

Steady-state ATR spectroscopy and spectroelectrochemistry were performed using a custom-built, broadband instrument described in previous publications, ${ }^{16,31}$ using ITO slides as planar waveguides. The mean tilt angle of the absorbance dipoles in PDI films was determined using polarized ATR spectroscopy, as described previously. ${ }^{15,16,32}$ ATR spectra acquired as a function of potential applied to the ITO slide (the working electrode) were measured using TM polarized light following procedures described previously. ${ }^{27}$

\section{PM-ATR spectroscopy}

Detailed descriptions of the PM-ATR instrument and the theory underlying PM-ATR and its use for determining ET rate constants for redox-active thin films have been published. ${ }^{16,31,33-35}$ Briefly, a sinusoidally modulated potential was applied to the PDI-modified ITO slide (the working electrode with 
an active area of $0.8 \mathrm{~cm}^{2}$ defined by a silicone gasket) centered around the midpoint potential $\left(E_{\mathrm{dc}}\right)$ for the redox couple. Collimated, TM polarized light was coupled into/out of the waveguide using two BK7 prisms positioned $20 \mathrm{~mm}$ apart. The internal reflection angle was $73-74^{\circ}$, which generated two total internal reflections at the $\mathrm{ITO} /$ solution interface. The $E_{\mathrm{dc}}$ was $-0.84 \mathrm{~V}$ vs. the $\mathrm{Ag} / \mathrm{AgNO}_{3}$ pseudo-reference electrode and the sinusoidally modulated voltage $\left(E_{\text {ac }}\right)$ was $20 \mathrm{mV}_{\mathrm{rms}}(0.028 \mathrm{~V}$ peak-to-peak). The in-phase $\left(\operatorname{Re}\left(R_{a c}\right)\right)$ and out-of-phase $\left(\operatorname{Im}\left(R_{a c}\right)\right)$ electroreflectance $(\mathrm{ER})$ signals were measured at $460 \mathrm{~nm}$ as a function of modulation frequency. The ET rate constant $\left(k_{\mathrm{s}, \mathrm{opt}}\right)$ was calculated from $k_{\mathrm{s}, \mathrm{opt}}=0.5 \omega^{2} R_{\mathrm{s}} C_{\mathrm{dl}}$, where $\omega$ is the frequency at which the reflectance signal is completely out of phase $\left(\operatorname{Re}\left(R_{a c}\right)=0\right), R_{\mathrm{s}}$ is the solution resistance, and $C_{\mathrm{dl}}$ is the double-layer capacitance. $R_{\mathrm{s}}$ and $C_{\mathrm{dl}}$ were measured by electrochemical impedance spectroscopy using an EG\&G Model 263A potentiostat/galvanostat coupled with a Model 1025 frequency response detector operated with PowerSuite software (Princeton Applied Research) in the same ATR spectroelectrochemical flow cell used for the PM-ATR measurements.

\section{Photoelectrochemistry}

Photoelectrochemical measurements were performed using the same three-electrode cell used for the CV measurements, with an ITO slide as the working electrode, a Pt wire as the counter electrode, and a $\mathrm{AgCl}$-coated silver wire $(\mathrm{Ag} / \mathrm{AgCl})$ as the pseudo-reference electrode. ${ }^{27}$ The $\mathrm{Ag} / \mathrm{AgCl}$ electrode was calibrated with the $\mathrm{Fc} / \mathrm{Fc}^{+}$redox couple for which the measured midpoint potential was $0.49 \mathrm{~V}$. The electrolyte solution was $0.1 \mathrm{M}$ TBAP in acetonitrile containing $500 \mu \mathrm{M}$ of aluminum phthalocyanine hydroxide (AlOHPc) as the electron donor. Additional experimental details are given in the previous publication. ${ }^{27}$ 


\section{RESULTS AND DISCUSSION}

\section{Synthesis of PDI-PA molecules}

Alkyl-PDI-PA was synthesized as previously described. ${ }^{27}$ The synthesis of the new PDI-PAs is shown in Scheme 1. The asymmetry necessary for the synthesis of these molecules was introduced by use of the perylene mixed diester / anhydride 1, which was prepared following a pathway reported by Xue et al. $;^{36}$ the reactivity difference between its anhydride and ester moieties is sufficiently large to allow nucleophilic attack by an amine to selectively take place at the anhydride. Thus, imidization of 1 with 2,6-dibromo-4-butylaniline, in the presence of imidazole and toluene at high temperatures, yielded the common intermediate 2. Typical imidization reactions are carried out at higher temperatures $(>180$ ${ }^{\circ} \mathrm{C}$ ) and with prolonged reaction times (up to $24 \mathrm{~h}$ ); under such conditions, for example, condensation of 2,6-diisopropyl aniline with perylene dianhydride yields the corresponding diimide in $>60 \%$ yield. ${ }^{37}$ However, debromination of $\mathbf{2}$ is observed at high temperature and so lower reaction temperatures, $130-140{ }^{\circ} \mathrm{C}$, were used here to limit this side reaction; the lower temperatures, and likely the deactivation of the amine by its bromo substituents, result in a relatively low isolated yield of $26 \%$. The boronic esters 1-GEN and 2-GEN, which were prepared following procedures by Ranta et $a l .{ }^{38}$ and Finke et al., ${ }^{39}$ respectively, were coupled to $\mathbf{2}$ via Suzuki coupling reactions to obtain $\mathbf{3}$ and $\mathbf{4}$ in moderate yields. Hydrolysis of intermediates $\mathbf{3}$ and $\mathbf{4}$ with $p$-toluenesulfonic acid gave the anhydrides $\mathbf{5}$ and 6, which, upon imidization with the appropriate aniline phosphonates, followed by ester hydrolysis with bromotrimethyl silane, yielded the desired PDIs.

\section{Electrochemical properties, molecular orientation, and surface coverage of PDI films}

The CVs of the four types of PDI-PA films deposited on ITO electrodes are shown in Figure 2. All 
showed the expected pair of one-electron reduction processes typical of PDIs in solution and similar to results presented in our previous study of PDI-PAs on ITO. ${ }^{31}$ The midpoint reduction potentials $\left(E^{0}\right)$ for the four types of films, listed in Table 1, are very similar and also are consistent with the literature data for dissolved PDIs, ${ }^{25,26}$ indicating that the redox thermodynamics of the PDI moiety are not significantly altered by immobilization on ITO or the introduction of phenyl, terphenyl, and/or diisopropyl substituents. However, the substituents did have a measurable effect on the cathodic peak width. The full width at half maxima (FWHM) of both peaks are listed in Table S1 and show that their width decreased as the size of the PDI increased. Thus a greater dispersion in reduction potentials is correlated with a greater degree of PDI intermolecular interactions, evidence for which is provided below.

The electroactive surface coverages $(\Gamma)$ for PDI-PA films, listed in Table 1, were estimated by voltammetric integration of both reduction peaks and assuming an overall two-electron reduction. A decrease in $\Gamma$ was observed as bulky substituents were added to the PDI core, which is qualitatively consistent with the expectation that the surface-projected area per adsorbed molecule will increase through the series of: alkyl-PDI-PA, (phenyl) $)_{2}$-PDI-PA, (terphenyl) $)_{2}$-PDI-PA $\approx\left(\right.$ terphenyl) ${ }_{2}$-PDI-IP-PA. 


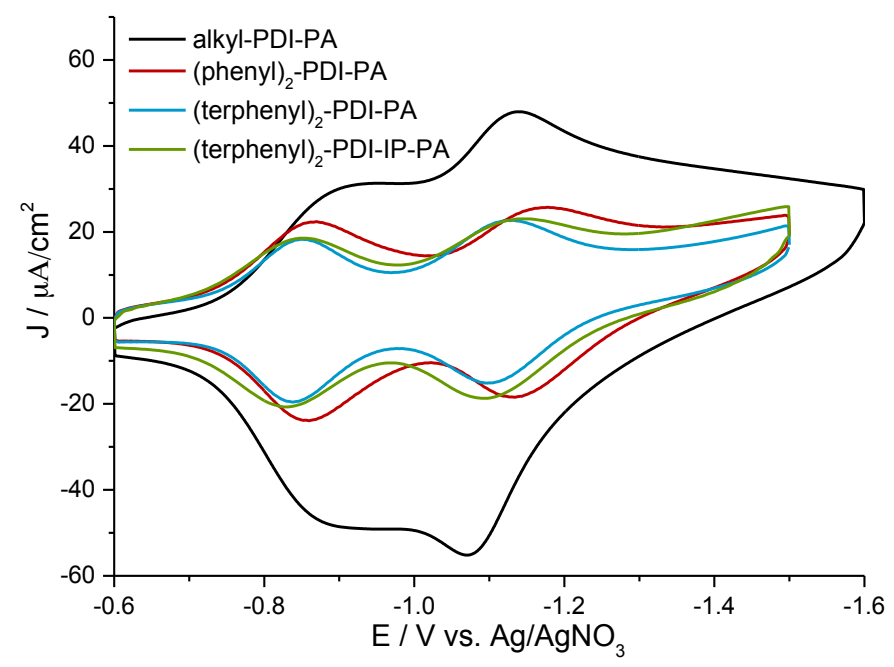

Figure 2. Representative cyclic voltammograms of four types of PDI-PA films on ITO electrodes. The electrolyte was $0.1 \mathrm{M}$

TBAP in acetonitrile, and the scan rate was $0.5 \mathrm{~V} / \mathrm{s}$. The substantially higher current for alkyl-PDI-PA relative to the other three films is due to the correspondingly higher electroactive surface coverage (Table 1).

Table 1. Electroactive surface coverage $(\Gamma)$, monolayer percentage, and reduction midpoint potentials $\left(E^{0}\right)$ for four types of PDI-PA films on ITO. ${ }^{\mathrm{a}}$

\section{alkyl-PDI-PA $^{\mathrm{b}} \quad(\text { phenyl })_{2}$-PDI-PA $\quad(\text { terphenyl })_{2}$-PDI-PA $\quad(\text { terphenyl })_{2}$-PDI-IP-PA}

\begin{tabular}{|c|c|c|c|c|}
\hline$\Gamma\left(10^{-11} \mathrm{~mol} / \mathrm{cm}^{2}\right)$ & $11 \pm 2$ & $4.2 \pm 0.3$ & $3.1 \pm 0.4$ & $3.2 \pm 0.2$ \\
\hline $\begin{array}{l}\text { Monolayer } \\
\text { percentage }\end{array}$ & $120 \pm 22 \%$ & $80 \pm 6 \%$ & $78 \pm 10 \%$ & $81 \pm 5 \%$ \\
\hline $\begin{array}{l}E^{0,}\left(1^{\text {st }}, \mathrm{V} \text { vs. }\right. \\
\left.\mathrm{Ag} / \mathrm{AgNO}_{3}\right)\end{array}$ & $-0.91 \pm 0.04 \mathrm{~V}$ & $-0.86 \pm 0.02 \mathrm{~V}$ & $-0.84 \pm 0.03 \mathrm{~V}$ & $-0.84 \pm 0.01 \mathrm{~V}$ \\
\hline $\begin{array}{l}E^{0,}\left(2^{\text {nd }}, \mathrm{V} \text { vs. }\right. \\
\left.\mathrm{Ag} / \mathrm{AgNO}_{3}\right)\end{array}$ & $-1.15 \pm 0.05 \mathrm{~V}$ & $-1.14 \pm 0.04 \mathrm{~V}$ & $-1.12 \pm 0.04 \mathrm{~V}$ & $1.13 \pm 0.03 \mathrm{~V}$ \\
\hline
\end{tabular}


Figure 3A shows TM-polarized and transverse electric- (TE-) polarized ATR spectra of the four types of PDI-PA films. Molecular orientation in these films was assessed from the dichroic ratio determined from integration of the TE- and TM-polarized absorbance bands (over 450-550 nm), and assuming that the PDI absorption transition dipole is aligned with the long molecular axis. ${ }^{40,41}$ Table 2 summarizes the mean tilt angles $(\theta)$ between the PDI long molecular axis and surface normal for the four types of films. The $\theta$ range, $33-42^{\circ}$, reflects a predominately out-of-plane orientation in all four films, which is consistent with the previous study of alkyl-PDI-PA and its biphenyl-bridged analogue. ${ }^{27}$ Previous studies ${ }^{11}$ of structurally simpler aryl-PAs (e.g., phenyl-PA) on ITO and indium zinc oxide have reported much smaller tilt angles $\left(<30^{\circ}\right)$. To our knowledge, only one other orientation study of an extended "rigid rod" aryl PA adsorbed to a TCO has been published. Gundlach et al. ${ }^{42}$ reported a tilt angle of $66^{\circ}$ for a perylene attached to $\mathrm{TiO}_{2}$ via a rigid, three-ring bridge with a PA anchor. Their result and ours suggest that relative to phenyl-PAs, PAs bearing larger aromatic ring systems adopt a more tilted orientation when bound to TCOs. 

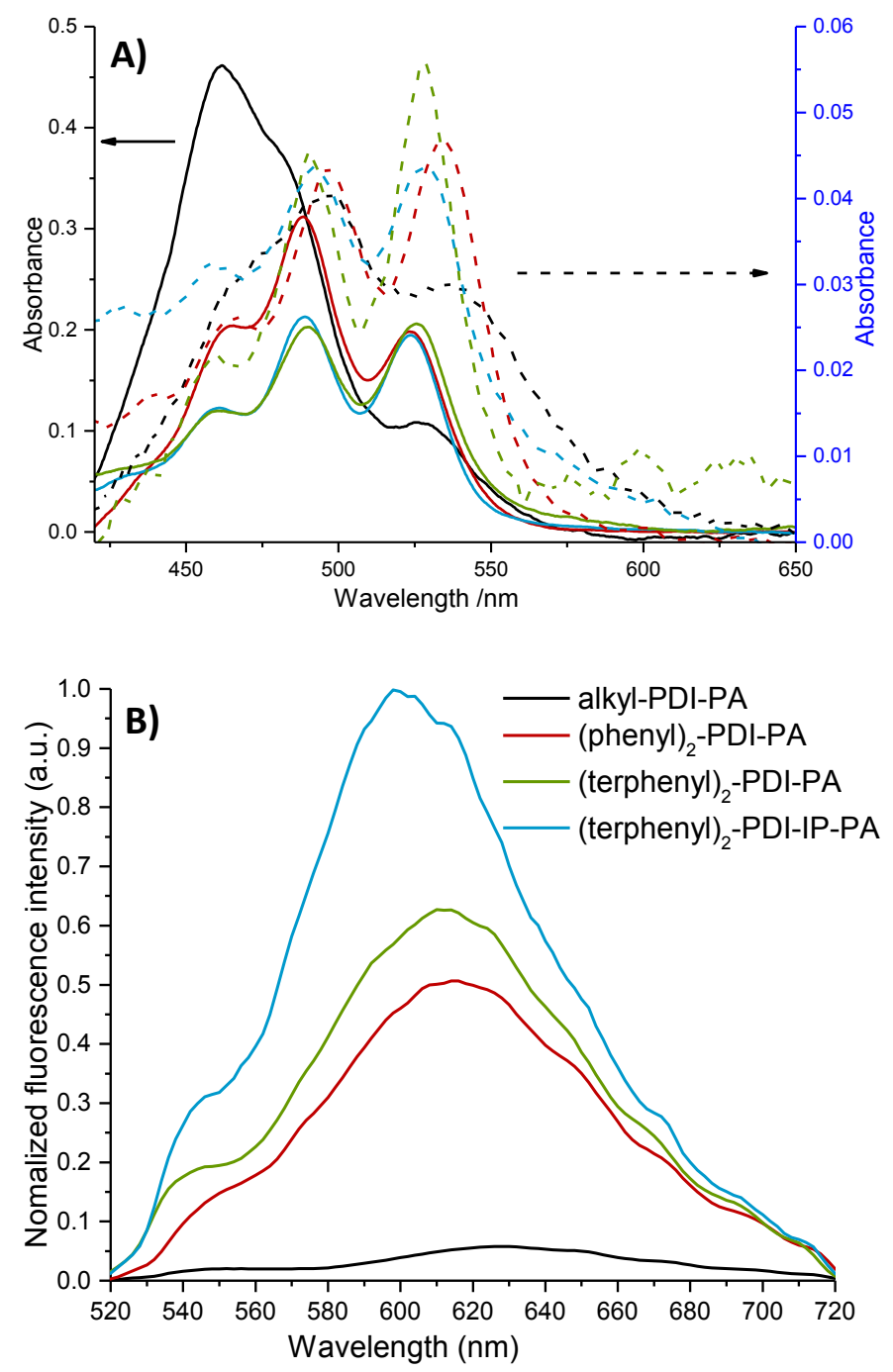

Figure 3. A) ATR spectra of PDI-PA films on ITO in TM (solid lines) and TE (dashed lines) polarizations: alkyl-PDI-PA (black lines), (phenyl) $)_{2}$-PDI-PA film (red lines), (terphenyl) $)_{2}$-PDI-PA (blue lines), and (terphenyl) 2 -PDI-IP-PA (green lines).

B) Normalized TIRF spectra of the four types of PDI-PA films on ITO. 
Table 2. Ratio of 0-0/0-1 absorbance bands $\left(\mathrm{A}_{0-0} / \mathrm{A}_{0-1}\right)$, tilt angle $(\theta)$, and ET rate constant $\left(k_{\mathrm{s}, \text { opt }}\right)$ for four types of PDI-PA films on ITO. ${ }^{\text {a }}$

\begin{tabular}{|c|c|c|c|c|}
\hline & alkyl-PDI-PA $^{\mathrm{c}}$ & (phenyl) $)_{2}$-PDI-PA & $(\text { terphenyl) })_{2}$-PDI-PA & (terphenyl) $)_{2}$-PDI-IP-PA \\
\hline $\mathbf{A}_{0-0} / \mathbf{A}_{0-1}{ }^{b}$ & $0.36 \pm 0.02$ & $0.62 \pm 0.04$ & $0.85 \pm 0.05$ & $0.98 \pm 0.03$ \\
\hline tilt angle $(\theta)$ & $33^{\circ} \pm 4^{\circ}$ & $38^{\circ} \pm 2^{\circ}$ & $41^{\circ} \pm 3^{\circ}$ & $42^{\circ} \pm 2^{\circ}$ \\
\hline$k_{\mathrm{s}, \mathrm{opt}}\left(\mathrm{s}^{-1}\right)^{\mathrm{d}}$ & $(1.4 \pm 0.3) \times 10^{4}$ & $(1.9 \pm 0.7) \times 10^{3}$ & $(1.2 \pm 0.1) \times 10^{3}$ & $(1.0 \pm 0.3) \times 10^{3}$ \\
\hline \multicolumn{5}{|c|}{${ }^{\mathrm{a}} n \geq 4$ for all measurements. } \\
\hline \multicolumn{5}{|c|}{${ }^{\mathrm{b}}$ Obtained from the TM-polarized ATR spectra. } \\
\hline${ }^{\mathrm{c}}$ From refer & ce 27 & & & \\
\hline
\end{tabular}

reduction reaction. The $R_{\mathrm{S}}$ and $C_{\mathrm{dl}}$ values used to calculate $k_{\mathrm{s}, \mathrm{opt}}$ for these films are listed in Table $\mathrm{S} 2$.

Table 2 also shows that $\theta$ increases as the degree of substitution increases, which is attributed to a difference in the degree of PDI aggregation. Cofacial $\pi$ - $\pi$ interactions among adjacent PDIs bound to ITO via phenyl-PA groups may favor a more out-of-plane orientation (i.e., more upright where presumably a higher degree of such interactions are achievable), and thus a smaller $\theta$ value. Conversely, bulky substituent groups disrupt these interactions; this is discussed further below.

The tilt angles were used in combination with MM2 energy minimization (Chembio 3D, PerkinElmer) to estimate the projected molecular area of each type of PDI modifier adsorbed to a planar surface via its PA group. The projected areas are: $180 \AA^{2} /$ molecule for alkyl-PDI-PA, $315 \AA^{2} / \mathrm{molecule}^{2}$

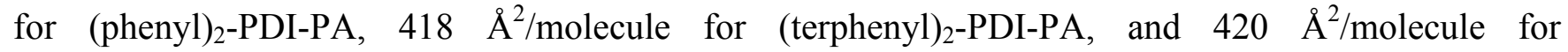
(terphenyl) $)_{2}$-PDI-IP-PA. The trend in these values is consistent with the 3.5 -fold variation in $\Gamma$ shown in 
Table 1. These projected area estimates along with the $\Gamma$ values were then used to estimate the percent monolayer coverages, also listed in Table 1 . The coverage is about one monolayer for all four PDI films. ${ }^{43}$

\section{Degree of aggregation in PDI films}

ATR and TIRF spectroscopies were used to characterize the degree of aggregation in the four types of PDI-PA films. PDI aggregation is known to cause broadening of the UV-visible absorbance bands and can be characterized by the ratio of the $0-0(c a .525 \mathrm{~nm})$ to the $0-1(c a .488 \mathrm{~nm})$ vibronic bands

within the $\mathrm{S}_{0}-\mathrm{S}_{1}$ manifold. ${ }^{44-47}$ A $0-0 / 0-1$ band ratio close to 1.6 is considered to be monomeric. ${ }^{45,48,49}$ Here a 0-0/0-1 band ratio of 1.6 was measured for all four PDI-PAs when dissolved in the solvents from which they were adsorbed to ITO (Figure S3 in SI), indicating they are essentially fully monomeric prior to film formation. Comparing the spectra in Figure 3A to the solution spectra shows that immobilization on ITO produced significant spectral changes, including band broadening, hypsochromic shifts, and decreases in the $0-0 / 0-1$ band ratios, all of which are indicative of $\mathrm{H}$-aggregate formation. ${ }^{45,50,51}$ Although aggregation is evident in all four film types, the degree varied. As increasingly bulky substituents were added to the PDI core, the vibronic bands became narrower and better resolved, and the $0-0$ band became more prominent. The $0-0 / 0-1$ band ratios extracted from the TM-polarized spectra (listed in Table 2; the TM data were used due to a higher signal to noise ratio) increased from 0.36 for alkyl-PDI-PA films to 0.98 for (terphenyl) 2 -PDI-IP-PA films, showing an inverse correlation between the size of the bulky substituents and the degree of aggregation.

TIRF spectra of the four types of PDI-PA films on ITO are shown in Figure 3B. The spectra were normalized to $\Gamma$ for each film and then, for comparison purposes, normalized to the highest intensity in 
the (terphenyl) $)_{2}$-PDI-PA spectrum. The spectra, therefore, reflect relative fluorescence quantum efficiencies. None of the films showed monomeric-like emission which is distinguished by three, well-resolved vibronic bands in the $500-650 \mathrm{~nm}$ regime; ${ }^{52-55}$ instead a featureless broad band at 550-800 $\mathrm{nm}$ that is characteristic of aggregation was observed in each case. However, as the size/number of substituents increased through the series of alkyl-PDI-PA, (phenyl) ${ }_{2}$-PDI-PA, (terphenyl) 2 -PDI-PA, and (terphenyl) $)_{2}$-PDI-IP-PA: a) the peak emission wavelength blue-shifted by $c a .0 .15 \mathrm{eV}$ (from $640 \mathrm{~nm}$ to $593 \mathrm{~nm}$ ); and b) the fluorescence quantum efficiency increased, which is indicative of a lower degree of self-quenching. Both the hypsochromic shift ${ }^{52,55}$ and the change in quantum yield ${ }^{44,47}$ are consistent with the UV-visible data and show that introduction of phenyl, terphenyl, and/or diisopropyl substituents to the PDI core markedly decreased aggregation in monolayer films of these molecules on ITO.

\section{Spectroelectrochemistry of PDI films}

TM-polarized ATR spectra of (terphenyl) $)_{2}$-PDI-IP-PA, (terphenyl) 2 -PDI-PA, and (phenyl) $)_{2}$ PDI-PA films acquired as a function of the potential applied to the ITO electrode are shown in the left panels of Figure S4. The spectroelectrochemical behavior of all three films was very similar to that of alkyl-PDI-PA presented in the previous paper. ${ }^{27}$ The PDI anion radical absorption bands appeared at wavelengths longer than $600 \mathrm{~nm}$ when the potential was stepped from $-0.6 \mathrm{~V}$ to $-1.1 \mathrm{~V} v s . \mathrm{Ag} / \mathrm{AgNO}_{3}$, while PDI dianion absorption bands arose in the 500-600 $\mathrm{nm}$ region at more negative potentials. The complete bleach of the neutral-state absorbance bands indicates that nearly all adsorbed PDI molecules in these films were electrochemically active; thus the $\Gamma$ values listed in Table 1 represent the total surface coverage for each film. The absorbance $v s$. applied potential at discrete wavelengths assigned to PDI $(460 \mathrm{~nm})$ and $\mathrm{PDI}^{2-}(569 \mathrm{~nm})^{56-58}$ is plotted in the right panels of Figure S4. Fitting these curves 
provided estimates of $E^{0}$ for the first and second reductions in each type of film. These values are listed in Table S3 and are consistent with the values determined by cyclic voltammetry.

\section{Electron-transfer kinetics at the PDI film/ITO interface}

ET rate constants for (terphenyl) $)_{2}$-PDI-IP-PA, (terphenyl) $)_{2}-\mathrm{PDI}-\mathrm{PA}$, and (phenyl) 2 -PDI-PA films on ITO were measured using PM-ATR at applied potentials corresponding to the first reduction reaction. Table 2 lists the $k_{\mathrm{s} \text {,opt }}$ values along with the rate constant for alkyl-PDI-PA films reported in the previous paper. $^{27}$ These data exhibit a clear trend: a higher degree of PDI aggregation resulted in a faster ET process. Three possible explanations for this trend are:

a) A more aggregated film has a higher extent of extended $\pi-\pi$ conjugation, and this may lower the reorganization energy for electron transfer. ${ }^{16,55,59,60}$ However, intermolecular coupling sufficiently strong to significantly reduce the reorganization energy might also be expected to be accompanied by a shift in redox potential; e.g., a phthalocyanine dimer is more readily oxidized than the corresponding monomer. ${ }^{16}$ The data in Tables 1 and S3 show that the $E^{0,}$ values for the four types of PDI molecules are statistically equivalent; thus it unlikely that differences in reorganization energy are the source of the difference in the $k_{\mathrm{s} \text {,opt }}$ values in Table 2 .

b) We assume that a through-bond pathway dominates tunneling between the PDI moiety and the ITO electrode, and thus the tunneling distance $(r)$ is the same for all four films. ${ }^{61}$ However, if direct through-space tunneling occurs, $r$ depends on $\theta$ and thus differences in $\theta$ could affect ET kinetics. ${ }^{15,16,27}$ To assess this possibility, $r$ values were estimated for $\theta$ values of $33^{\circ}$ and $42^{\circ}$ which are the respective mean tilt angles for alkyl-PDI-PA and (terphenyl) ${ }_{2}$-PDI-IP-PA films (Table 2). Standard ET rate constants $\left(k_{\Gamma, \mathrm{s}}\right)$ were then calculated from $k_{\Gamma, \mathrm{s}}=k_{0} e^{-\beta_{r} r}$, where $\beta_{\mathrm{r}}$ is the exponential decay 
coefficient, assumed here to be $0.28 \AA^{-1},{ }^{27}$ and $k_{0}$ is the extrapolated value of the rate constant for $r=$ $0 .^{62}$ This analysis predicts a 1.1-fold increase in the rate constant when the tilt angle is increased from $33^{\circ}$ to $42^{\circ}$. The opposite trend was observed here: $k_{\mathrm{s}, \mathrm{opt}}$ for (terphenyl) 2 -PDI-IP-PA was 14 -fold less than $k_{\mathrm{s}, \mathrm{opt}}$ for alkyl-PDI-PA (Table 2). Thus the differences in molecular orientation among these films are not the cause of the differences in ET kinetics.

c) In a more highly aggregated film, the spacing between adjacent PDI molecular cores is smaller, which is anticipated to enhance the rate of intermolecular electron self-exchange between surface-bound PDI $^{\circ-}$ and PDI species. ${ }^{63,64}$ Studies of the conductivity of air-plasma etched ITO show that the surface is quite heterogeneous and electrical 'hot spots' are discernible. ${ }^{65}$ MacDonald et al. reported that these electrical hot spots also are detectable when a $20 \mathrm{~nm}$ thick layer of copper phthalocyanine $(\mathrm{CuPc})$ is deposited on ITO ${ }^{66}$ These studies suggest that the PDI/ITO ET reaction will be much faster at those hotspots. For PDI-PAs not directly bound at hot spots, direct electron exchange with the ITO will be much slower, and the dominant mechanism for ET is likely to involve both lateral self exchange (PDI/PDI ${ }^{-}$transfer) and exchange between a PDI at a hot spot and ITO. Accordingly we hypothesize that the differences in observed rate constants reflect differences in the rates of intermolecular electron self-exchange. This hypothesis is consistent with a number of studies showing that the rate of lateral electron self-exchange in redox-active films on TCOs is enhanced as the surface coverage of the modifier is increased. ${ }^{67-70}$ Although it provides a reasonable explanation for the data, this hypothesis remains to be tested and will be the subject of future studies. It also suggests that the intermolecular electron self-exchange rate in alkyl-PDI-PA films might be further increased by removing the C11 chains to increase the degree of aggregation. Removing the chains, however, would make this molecule 
less soluble (possibly insoluble) ${ }^{27}$ and hence incompatible with adsorption from solution.

\section{Photoelectrochemical measurements of PDI films}

To assess PDI modifier films as "ET mediator" layers, photoelectrochemical measurements were performed using dissolved AlOHPc as an electron donor. An energy level diagram of the photoelectrochemical cell is presented in the SI of the previous paper. ${ }^{27}$ Electron transfer to the PDI-PA film was photo-initiated by excitation of AlOHPc at $632.8 \mathrm{~nm}$, a wavelength at which PDI does not absorb. Figure S5 shows an example of the photocurrent measured as a function of applied potential for a (terphenyl) $)_{2}$-PDI-PA-modified ITO electrode. Based on the results from scans of photocurrent vs. applied potential, a holding potential of $0.6 \mathrm{~V} v s . \mathrm{Ag} / \mathrm{AgCl}(-5.2 \mathrm{eV} v s$. vacuum) was selected for the photocurrent measurements.

Previously we showed that coating the ITO electrode with a alkyl-PDI-PA film increased the photocurrent $c a$. 7-fold relative to bare ITO. Here similar behavior was observed for the films formed from the three bulkier PDI-PAs (Figure 4). The enhancements in photocurrent were $c a$. 4- to 7-fold $v s$. bare ITO bare, with the variations in this range being statistically insignificant. Control experiments showed that AlOHPc adsorption on bare ITO and PDI-modified ITO electrodes was minimal. These results demonstrate that PDI acts as an ET mediator between AlOHPc and ITO, regardless of the extent of aggregation. 


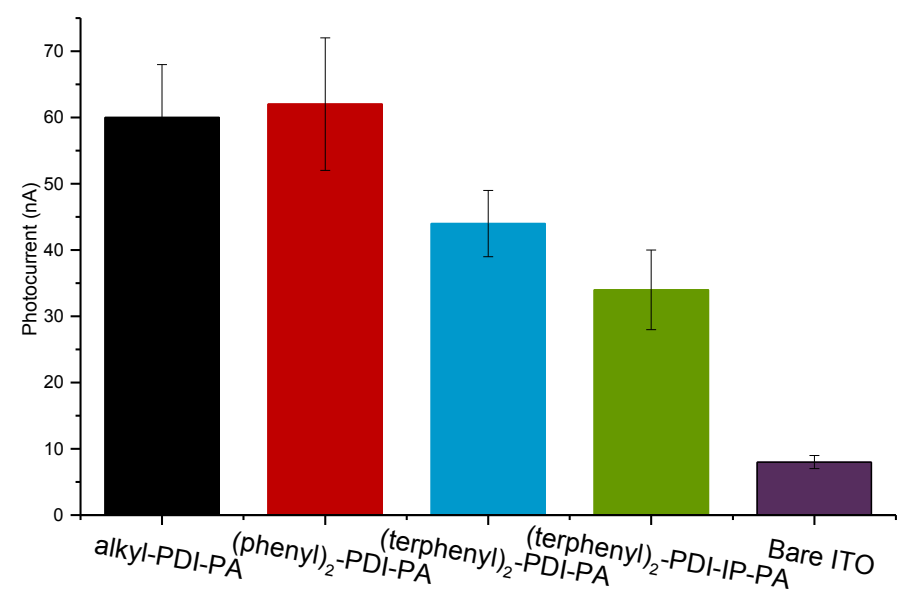

Figure 4. Photocurrent produced by the four PDI-modified electrodes and bare ITO, obtained from photoelectrochemical measurements as described in the text. Error bars indicate one standard deviation. The data for alkyl-PDI-PA and bare ITO are from the previous paper. ${ }^{27}$

\section{CONCLUSIONS}

The effects of molecular aggregation in PDI-PA monolayer films on ITO on ET reactions across PDI/ITO interfaces was investigated. By introducing bulky substituents at the imide position of PDI, aggregation was systematically reduced without changing the electronic properties or significantly altering the molecular orientation. ET rate constants decreased as the degree of PDI aggregation was reduced, which is hypothesized to be due to a lower rate of electron self-exchange rate between PDI molecules. Photoelectrochemical measurements showed that modification of ITO electrodes with PDI-PA films enhanced the electron collection efficiency across the AlOHPc/ITO interface, which is attributed to the PDI-PA film acting as an ET mediator. Overall, these results show how structural variables can be tuned to control ET kinetics at redox-active organic/ITO interfaces, and they should aid future efforts to molecularly engineer these types of interfaces for use in organic electronic devices. 


\section{SUPPORTING INFORMATION AVAILABLE}

Detailed synthesis procedures, NMR spectra, HPLC purification procedures and results, spectral, electrochemical, and impedance characterization of PDI-PA films, absorbance spectra of dissolved PDIs, and photoelectrochemical data. This material is available free of charge via the Internet at http://pubs.acs.org.

\section{ACKNOWLDGEMENTS}

This research was partially supported as part of the Center for Interface Science: Solar-Electric Materials (CIS:SEM), an Energy Frontier Research Center funded by the U.S. Department of Energy, Office of Science, Office of Basic Energy Sciences under Award No. DE-SC0001084. This material is based upon work that was partially supported by the National Science Foundation under Grant No.

DMR-1506504. The research conducted at the Georgia Institute of Technology was also supported by the Department of the Navy, Office of Naval Research through Award No. N00014-14-1-0580 / N00014-16-1-2520 (CAOP MURI).

\section{REFERENCES}

(1) Khodabakhsh, S.; Poplavskyy, D.; Heutz, S.; Nelson, J.; Bradley, D. D. C.; Murata, F.; Jones, T. S. Using Self-Assembling Dipole Molecules to Improve Hole Injection in Conjugated Polymers. Adv. Funct. Mater. 2004, 14, 1205-1210.

(2) Khodabakhsh, S.; Sanderson, B. M.; Nelson, J.; Jones, T. S. Using Self-Assembling Dipole Molecules to Improve Charge Collection in Molecular Solar Cells. Adv. Funct. Mater. 2006, 16, 95-100.

(3) Hau, S. K.; Yip, H.-L.; Acton, O.; Baek, N. S.; Ma, H.; Jen, A. K. Y. Interfacial Modification to Improve Inverted Polymer Solar Cells. J. Mater. Chem. 2008, 18, 5113-5119. 
(4) Song, C. K.; Luck, K. A.; Zhou, N.; Zeng, L.; Heitzer, H. M.; Manley, E. F.; Goldman, S.; Chen, L. X.; Ratner, M. A.; Bedzyk, M. J.; Chang, R. P. H.; Hersam, M. C.; Marks, T. J. "Supersaturated” Self-Assembled Charge-Selective Interfacial Layers for Organic Solar Cells. J. Am. Chem. Soc. 2014, 136, 17762-17773.

(5) DiBenedetto, S. A.; Facchetti, A.; Ratner, M. A.; Marks, T. J. Molecular Self-Assembled Monolayers and Multilayers for Organic and Unconventional Inorganic Thin-Film Transistor Applications. Adv. Mater. 2009, 21, 1407-1433.

(6) Hanson, E. L.; Guo, J.; Koch, N.; Schwartz, J.; Bernasek, S. L. Advanced Surface Modification of Indium Tin Oxide for Improved Charge Injection in Organic Devices. J. Am. Chem. Soc. 2005, 127, 10058-10062.

(7) Huang, Q.; Li, J.; Evmenenko, G. A.; Dutta, P.; Marks, T. J. Systematic Investigation of Nanoscale Adsorbate Effects at Organic Light-Emitting Diode Interfaces. Interfacial Structure-Charge Injection-Luminance Relationships. Chem. Mater. 2006, 18, 2431-2442.

(8) Xu, X.; Zhang, H.; Cao, K.; Cui, J.; Lu, J.; Zeng, X.; Shen, Y.; Wang, M. Lead Methylammonium Triiodide Perovskite-Based Solar Cells: An Interfacial Charge-Transfer Investigation. ChemSusChem 2014, 7 , 3088-3094.

(9) Armstrong, N. R.; Carter, C.; Donley, C.; Simmonds, A.; Lee, P.; Brumbach, M.; Kippelen, B.; Domercq, B.; Yoo, S. Interface Modification of ITO Thin Films: Organic Photovoltaic Cells. Thin Solid Films 2003, 445, 342-352.

(10) Armstrong, N. R.; Veneman, P. A.; Ratcliff, E.; Placencia, D.; Brumbach, M. Oxide Contacts in Organic Photovoltaics: Characterization and Control of Near-Surface Composition in Indium-Tin Oxide (ITO) Electrodes. Acc. Chem. Res. 2009, 42, 1748-1757.

(11) Paniagua, S. A.; Giordano, A. J.; Smith, O. N. L.; Barlow, S.; Li, H.; Armstrong, N. R.; Pemberton, J. E.; Brédas, J.-L.; Ginger, D.; Marder, S. R. Phosphonic Acids for Interfacial Engineering of Transparent Conductive Oxides. Chem. Rev. 2016, 116, 7117-7158.

(12) Hotchkiss, P. J.; Jones, S. C.; Paniagua, S. A.; Sharma, A.; Kippelen, B.; Armstrong, N. R.; Marder, S. R. The Modification of Indium Tin Oxide with Phosphonic Acids: Mechanism of Binding, Tuning of Surface Properties, and Potential for Use in Organic Electronic Applications. Acc. Chem. Res. 2011, 45, 337-346.

(13) Hotchkiss, P. J.; Li, H.; Paramonov, P. B.; Paniagua, S. A.; Jones, S. C.; Armstrong, N. R.; Brédas, J.-L.; Marder, S. R. Modification of the Surface Properties of Indium Tin Oxide with Benzylphosphonic Acids: A Joint Experimental and Theoretical Study. Adv. Mater. 2009, 21, 4496-4501.

(14) Chen, M.-C.; Chiou, Y.-S.; Chiu, J.-M.; Tedla, A.; Tai, Y. Marked Improvement in the Stability of Small Molecule Organic Photovoltaics by Interfacial Modification Using Self-Assembled Monolayers to Prevent Indium Diffusion into the Active Layer. J. Mater. Chem. A 2013, 1, 3680-3687.

(15) Lin, H.-C.; MacDonald, G. A.; Shi, Y.; Polaske, N. W.; McGrath, D. V.; Marder, S. R.; Armstrong, N. R.; Ratcliff, E. L.; Saavedra, S. S. Influence of Molecular Orientation on Charge-Transfer Processes at Phthalocyanine/Metal Oxide Interfaces and Relationship to Organic Photovoltaic Performance. J. Phys. Chem. C 2015, 119, 10304-10313.

(16) Lin, H. C.; Polaske, N. W.; Oquendo, L. E.; Gliboff, M.; Knesting, K. M.; Nordlund, D.; Ginger, D. S.; Ratcliff, E. L.; Beam, B. M.; Armstrong, N. R.; McGrath, D. V.; Saavedra, S. S. Electron-Transfer Processes in Zinc Phthalocyanine Phosphonic Acid Monolayers on ITO: Characterization of Orientation and Charge-Transfer Kinetics by Waveguide Spectroelectrochemistry. J. Phys. Chem. Lett. 2012, 3, 1154-1158. 
(17) Spiller, W.; Kliesch, H.; Wöhrle, D.; Hackbarth, S.; Röder, B.; Schnurpfeil, G. Singlet Oxygen Quantum Yields of Different Photosensitizers in Polar Solvents and Micellar Solutions. J. Porphyrins Phthalocyanines 1998, 2, 145-158.

(18) Khazraji, A. C.; Hotchandani, S.; Das, S.; Kamat, P. V. Controlling Dye (Merocyanine-540) Aggregation on Nanostructured $\mathrm{TiO}_{2}$ Films. An Organized Assembly Approach for Enhancing the Efficiency of Photosensitization. J. Phys. Chem. B 1999, 103, 4693-4700.

(19) He, J.; Benkö, G.; Korodi, F.; Polívka, T.; Lomoth, R.; Åkermark, B.; Sun, L.; Hagfeldt, A.; Sundström, V. Modified Phthalocyanines for Efficient Near-IR Sensitization of Nanostructured $\mathrm{TiO}_{2}$ Electrode. J. Am. Chem. Soc. 2002, 124, 4922-4932.

(20) Shallcross, R. C.; D’Ambruoso, G. D.; Pyun, J.; Armstrong, N. R. Photoelectrochemical Processes in Polymer-Tethered CdSe Nanocrystals. J. Am. Chem. Soc. 2010, 132, 2622-2632.

(21) Rangan, S.; Coh, S.; Bartynski, R. A.; Chitre, K. P.; Galoppini, E.; Jaye, C.; Fischer, D. Energy Alignment, Molecular Packing, and Electronic Pathways: Zinc(II) Tetraphenylporphyrin Derivatives Adsorbed on $\mathrm{TiO}_{2}(110)$ and $\mathrm{ZnO}(11-20)$ Surfaces. J. Phys. Chem. C 2012, 116, 23921-23930.

(22) Shin, W. S.; Jeong, H.-H.; Kim, M.-K.; Jin, S.-H.; Kim, M.-R.; Lee, J.-K.; Lee, J. W.; Gal, Y.-S. Effects of Functional Groups at Perylene Diimide Derivatives on Organic Photovoltaic Device Application. J. Mater. Chem. 2006, 16, 384-390.

(23) Oh, S. H.; Kim, B. G.; Yun, S. J.; Maheswara, M.; Kim, K.; Do, J. Y. The Synthesis of Symmetric and Asymmetric Perylene Derivatives and Their Optical Properties. Dyes Pigm. 2010, 85, 37-42.

(24) Sang Kwon Lee; Yanbing Zu; Andreas Herrmann; Yves Geerts; Klaus Mullen; Bard, A. J. Electrochemistry, Spectroscopy and Electrogenerated Chemiluminescence of Perylene, Terrylene, and Quaterrylene Diimides in Aprotic Solution. J. Am. Chem. Soc. 1999, 121, 3513-3520.

(25) Würthner, F. Perylene Bisimide Dyes as Versatile Building Blocks for Functional Supramolecular Architectures. Chem. Commun. 2004, 1564-1579.

(26) Huang, C.; Barlow, S.; Marder, S. R. Perylene-3,4,9,10-tetracarboxylic Acid Diimides: Synthesis, Physical Properties, and Use in Organic Electronics. J. Org. Chem. 2011, 76, 2386-2407.

(27) Zheng, Y.; Giordano, A. J.; Shallcross, R. C.; Fleming, S. R.; Armstrong, N. R.; Marder, S. R.; Saavedra, S. S. Surface Modification of Indium-Tin Oxide with Functionalized Perylene Diimide Molecules: Characterization of Orientation, Charge-Transfer Kinetics and Electronic Structure. J. Phys. Chem. C 2016, 120, 20040-20048.

(28) Lin, M.-J.; Jimenez, A. J.; Burschka, C.; Würthner, F. Bay-Substituted Perylene Bisimide Dye with an Undistorted Planar Scaffold and Outstanding Solid State Fluorescence Properties. Chem. Commun. 2012, 48, 12050-12052.

(29) Jimenez, A. J.; Lin, M.-J.; Burschka, C.; Becker, J.; Settels, V.; Engels, B.; Würthner, F. Structure-Property Relationships for 1,7-Diphenoxy-perylene Bisimides in Solution and in the Solid State. Chem. Sci. 2014, 5, 608-619.

(30) Cardona, C. M.; Li, W.; Kaifer, A. E.; Stockdale, D.; Bazan, G. C. Electrochemical Considerations for Determining Absolute Frontier Orbital Energy Levels of Conjugated Polymers for Solar Cell Applications. Adv. Mater. 2011, 23, 2367-2371.

(31) Doherty, W. J.; Donley, C. L.; Armstrong, N. R.; Saavedra, S. S. Broadband Spectroelectrochemical Attenuated Total Reflectance Instrument For Molecular Adlayer Studies. Appl. Spectrosc. 2002, 56, 920-927. 
(32) Mendes, S. B.; Bradshaw, J. T.; Saavedra, S. S. Technique for Determining the Angular Orientation of Molecules Bound to the Surface of an Arbitrary Planar Optical Waveguide. Appl. Opt. 2004, 43, 70-78.

(33) Feng, Z. Q.; Sagara, T.; Niki, K. Application of Potential-Modulated UV-Visible Reflectance Spectroscopy to Electron Transfer Rate Measurements for Adsorbed Species on Electrode Surfaces. Anal. Chem. 1995, 67, 3564-3570.

(34) Doherty, W. J.; Wysocki, R. J.; Armstrong, N. R.; Saavedra, S. S. Potential-Modulated, Attenuated Total Reflectance Spectroscopy of Poly(3,4-ethylenedioxythiophene) and Poly(3,4-ethylenedioxythiophene Methanol) Copolymer Films on Indium-Tin Oxide. J. Phys. Chem. B 2006, 110, 4900-4907.

(35) Araci, Z. O.; Runge, A. F.; Do Herty, W. J.; Saavedra, S. S. Correlating Molecular Orientation Distributions and Electrochemical Kinetics in Subpopulations of an Immobilized Protein Film. J. Am. Chem. Soc. 2008, 130, 1572-1573.

(36) Xue, C.; Sun, R.; Annab, R.; Abadi, D.; Jin, S. Perylene Monoanhydride Diester: A Versatile Intermediate for the Synthesis of Unsymmetrically Substituted Perylene Tetracarboxylic Derivatives. Tetrahedron Lett. 2009, 50, 853-856.

(37) Ramanan, C.; Smeigh, A. L.; Anthony, J. E.; Marks, T. J.; Wasielewski, M. R. Competition between Singlet Fission and Charge Separation in Solution-Processed Blend Films of 6,13-Bis(triisopropylsilylethynyl)pentacene with Sterically-Encumbered Perylene-3,4:9,10-bis(dicarboximide)s. $J$. Am. Chem. Soc. 2012, 134, 386-397.

(38) Ranta, J.; Kumpulainen, T.; Lemmetyinen, H.; Efimov, A. Synthesis and Characterization of Monoisomeric 1,8,15,22-Substituted $\left(\mathrm{A}_{3} \mathrm{~B}\right.$ and $\left.\mathrm{A}_{2} \mathrm{~B}_{2}\right)$ Phthalocyanines and Phthalocyanine-Fullerene Dyads. $J$. Org. Chem. 2010, 75, 5178-5194.

(39) Finke, A. D.; Moore, J. S. Iterative Synthesis of 1,3,5-Polyphenylene Dendrons via C-H Activation. Org. Lett. 2008, 10, 4851-4854.

(40) Christensen, R. L.; Drake, R. C.; Phillips, D. Time-Resolved Fluorescence Anisotropy of Perylene. J. Phys. Chem. 1986, 90, 5960-5967.

(41) Mizuguchi, J. Polymorph of N,N'-di-n-butylperylene-3,4:9,10-bis(dicarboximide) and Their Electronic Structure. Dyes Pigm. 2006, 70, 226-231.

(42) Gundlach, L.; Szarko, J.; Socaciu-Siebert, L. D.; Neubauer, A.; Ernstorfer, R.; Willig, F. Different Orientations of Large Rigid Organic Chromophores at the Rutile $\mathrm{TiO}_{2}$ Surface Controlled by Different Binding Geometries of Specific Anchor Groups. Phys. Rev. B 2007, 75, 125320.

(43) The value of $120 \%$ for alkyl-PDI-PA suggests a slightly higher coverage relative to the other three films, possibly indicative of partial multilayer formation. This cannot be stated definitively, however, given the respective standard deviation of $22 \%$ and the uncertainty in the projected molecular area calculation.

(44) Zhao, Q.; Zhang, S.; Liu, Y.; Mei, J.; Chen, S.; Lu, P.; Qin, A.; Ma, Y.; Sun, J. Z.; Tang, B. Z. Tetraphenylethenyl-Modified Perylene Bisimide: Aggregation-Induced Red Emission, Electrochemical Properties and Ordered Microstructures. J. Mater. Chem. 2012, 22, 7387.

(45) Liu, Y.; Wang, K.-R.; Guo, D.-S.; Jiang, B.-P. Supramolecular Assembly of Perylene Bisimide with $\beta$-Cyclodextrin Grafts as a Solid-State Fluorescence Sensor for Vapor Detection. Adv. Funct. Mater. 2009, 19 , 2230-2235.

(46) Jiang, B. P.; Guo, D. S.; Liu, Y. Self-Assembly of Amphiphilic Perylene-Cyclodextrin Conjugate and Vapor Sensing for Organic Amines. J. Org. Chem. 2010, 75, 7258-7264. 
(47) Chen, Z.; Baumeister, U.; Tschierske, C.; Würthner, F. Effect of Core Twisting on Self-Assembly and Optical Properties of Perylene Bisimide Dyes in Solution and Columnar Liquid Crystalline Phases. Chem. Eur. J. 2007, 13, 450-465.

(48) Li, A. D. Q.; Wang, W.; Wang, L. Q. Folding versus Self-Assembling. Chem. Eur. J. 2003, 9, 4594-4601.

(49) Wang, W.; Li, L.-S.; Helms, G.; Zhou, H.-H.; Li, A. D. Q. To Fold or to Assemble? J. Am. Chem. Soc. 2003, 125, 1120-1121.

(50) Yoo, H.; Yang, J.; Yousef, A.; Wasielewski, M. R.; Kim, D. Excimer Formation Dynamics of Intramolecular $\pi$-Stacked Perylenediimides Probed by Single-Molecule Fluorescence Spectroscopy. J. Am. Chem. Soc. 2010, 132, 3939-3944.

(51) Würthner, F.; Saha-Möller, C. R.; Fimmel, B.; Ogi, S.; Leowanawat, P.; Schmidt, D. Perylene Bisimide Dye Assemblies as Archetype Functional Supramolecular Materials. Chem. Rev. 2016, 116, 962-1052.

(52) Back, A.; Alloway, D.; Schlettwein, D.; Schilling, B.; Wang, J.-F.; Carducci, M.; Armstrong, N. R. Ordered Thin Films of Perylenetetracarboxylicdianhydride-bisimide and bis-(N-alkyl)-Quinacridone Dyes. Mat. Res. Soc. Symp. Proc. 2000, 620, M.3.2.1.

(53) Würthner, F.; Thalacker, C.; Diele, S.; Tschierske, C. Fluorescent J-Type Aggregates and Thermotropic Columnar Mesophases of Perylene Bisimide Dyes. Chem. Eur. J. 2001, 7, 2245-2253.

(54) Seibt, J.; Marquetand, P.; Engel, V.; Chen, Z.; Dehrn, V.; Würthner, F. On the Geometry Dependence of Molecular Dimer Spectra with an Application to Aggregates of Perylene Bisimide. Chem. Phys. 2006, 328, 354-362.

(55) Chen, Z.; Stepanenko, V.; Dehm, V.; Prins, P.; Siebbeles, L. D. A.; Seibt, J.; Marquetand, P.; Engel, V.; Würthner, F. Photoluminescence and Conductivity of Self-Assembled $\pi-\pi$ Stacks of Perylene Bisimide Dyes. Chem. Eur. J. 2007, 13, 436-449.

(56) Marcon, R. O.; Brochsztain, S. Aggregation of 3,4,9,10-Perylenediimide Radical Anions and Dianions Generated by Reduction with Dithionite in Aqueous Solutions. J. Phys. Chem. A 2009, 113, 1747-1752.

(57) Ahrens, M. J.; Fuller, M. J.; Wasielewski, M. R. Cyanated Perylene-3,4-dicarboximides and Perylene-3,4:9,10-bis(dicarboximide): Facile Chromophoric Oxidants for Organic Photonics and Electronics. Chem. Mater. 2003, 15, 2684-2686.

(58) Gosztola, D.; Niemczyk, M. P.; Svec, W.; Lukas, A. S.; Wasielewski, M. R. Excited Doublet States of Electrochemically Generated Aromatic Imide and Diimide Radical Anions. J. Phys. Chem. A 2000, 104, 6545-6551.

(59) Norton, J. E.; Brédas, J.-L. Theoretical Characterization of Titanyl Phthalocyanine as a P-Type Organic Semiconductor: Short Intermolecular $\pi-\pi$ Interactions Yield Large Electronic Couplings and Hole Transport Bandwidths. J. Chem. Phys. 2008, 128, 034701.

(60) Geng, Y.; Wang, J.; Wu, S.; Li, H.; Yu, F.; Yang, G.; Gao, H.; Su, Z. Theoretical Discussions on Electron Transport Properties of Perylene Bisimide Derivatives with Different Molecular Packings and Intermolecular Interactions. J. Mater. Chem. 2011, 21, 134-143.

(61) Salomon, A.; Cahen, D.; Lindsay, S.; Tomfohr, J.; Engelkes, V. B.; Frisbie, C. D. Comparison of Electronic Transport Measurements on Organic Molecules. Adv. Mater. 2003, 15, 1881-1890.

(62) Smalley, J. F.; Feldberg, S. W.; Chidsey, C. E. D.; Linford, M. R.; Newton, M. D.; Liu, Y. P. The Kinetics of Electron-Transfer Through Ferrocene-Terminated Alkanethiol Monolayers on Gold. J. Phys. Chem. 
1995, 99, 13141-13149.

(63) George, C. B.; Szleifer, I.; Ratner, M. A. Lateral Electron Transport in Monolayers of Short Chains at Interfaces: A Monte Carlo Study. Chem. Phys. 2010, 375, 503-507.

(64) Forster, R. J.; Keyes, T. E.; Majda, M. Homogeneous and Heterogeneous Electron Transfer Dynamics of Osmium-Containing Monolayers at the Air/Water Interface. J. Phys. Chem. B 2000, 104, 4425-4432.

(65) Brumbach, M.; Veneman, P. A.; Marrikar, F. S.; Schulmeyer, T.; Simmonds, A.; Xia, W.; Lee, P.; Armstrong, N. R. Surface Composition and Electrical and Electrochemical Properties of Freshly Deposited and Acid-Etched Indium Tin Oxide Electrodes. Langmuir 2007, 23, 11089-11099.

(66) MacDonald, G. A.; Veneman, P. A.; Placencia, D.; Armstrong, N. R. Electrical Property Heterogeneity at Transparent Conductive Oxide/Organic Semiconductor Interfaces: Mapping Contact Ohmicity Using Conducting-Tip Atomic Force Microscopy. ACS Nano 2012, 6, 9623-9636.

(67) The apparent lateral diffusion coefficient for lateral charge transfer has been shown to exhibit classical percolation behavior, where a large increase in self-exchange occurs when the charge-transport percolation threshold is exceeded, which typically occurs at about $50 \%$ of monolayer coverage. ${ }^{68-70}$ Here, a comparison of the surface coverage data (Table 1) and the $\mathrm{k}_{\mathrm{s}, \mathrm{opt}}$ data (Table 2) suggest that the much higher rate constant measured for alkyl-PDI-PA films could be due to percolation, however additional data at intermediate surface coverages would be required to make a definitive statement.

(68) Papageorgiou, N.; Grätzel, M.; Enger, O.; Bonifazi, D.; Diederich, F. Lateral Electron Transport inside a Monolayer of Derivatized Fullerenes Anchored on Nanocrystalline Metal Oxide Films. J. Phys. Chem. B 2002, 106, 3813-3822.

(69) Bonhôte, P.; Gogniat, E.; Tingry, S.; Barbé, C.; Vlachopoulos, N.; Lenzmann, F.; Comte, P.; Grätzel, M. Efficient Lateral Electron Transport inside a Monolayer of Aromatic Amines Anchored on Nanocrystalline Metal Oxide Films. J. Phys. Chem. B 1998, 102, 1498-1507.

(70) Magoga, M.; Joachim, C. Conductance and Transparence of Long Molecular Wires. Phys. Rev. B 1997, $56,4722-4729$. 
TOC GRAPHIC

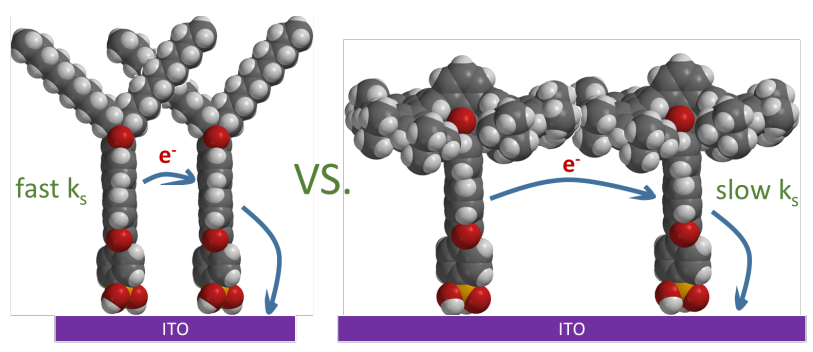

